OPEN ACCESS

Edited by:

Elka R. Georgieva

Cornell University, USA

Reviewed by:

Boris Martinac

Victor Chang Cardiac Research

Institute, Australia

Marco Bortolus,

University of Padova, Italy

*Correspondence:

Martina Huber

huber@physics.leidenuniv.n

Armagan Kocer

a.kocer@umcg.n

${ }^{\dagger}$ Present Address:

Anna Dimitrova,

Faculty of Mathematics and Natural

Sciences, Institute of Physics,

Technical Physics I, TU IImenau,

IImenau, Germany;

Martin Walko,

Faculty of Science, Institute of Chemistry, P. J. Safarik University in

Kosice, Kosice, Slovakia

Specialty section:

This article was submitted to Membrane Physiology and Membrane

Biophysics,

a section of the journal

Frontiers in Physiology

Received: 04 July 2016

Accepted: 30 August 2016 Published: 21 September 2016

Citation:

Dimitrova A, Walko M, Hashem

Shabestari M, Kumar P, Huber $M$ and Kocer A (2016) In situ, Reversible Gating of a Mechanosensitive Ion

Channel through Protein-Lipid Interactions. Front. Physiol. 7:409.

doi: 10.3389/fphys.2016.00409

\section{In situ, Reversible Gating of a Mechanosensitive Ion Channel through Protein-Lipid Interactions}

\author{
Anna Dimitrova ${ }^{1 \dagger}$, Martin Walko ${ }^{1 \dagger}$, Maryam Hashemi Shabestari ${ }^{2}$, Pravin Kumar ${ }^{2}$, \\ Martina Huber ${ }^{2 *}$ and Armagan Kocer ${ }^{3 *}$
}

${ }^{1}$ Department of Biochemistry, University of Groningen, Groningen, Netherlands, ${ }^{2}$ Huygens-Kamerlingh Onnes Laboratory, Department of Physics, Leiden University, Leiden, Netherlands, ${ }^{3}$ Neuroscience Department, University of Groningen, University Medical Center Groningen, Groningen, Netherlands

Understanding the functioning of ion channels, as well as utilizing their properties for biochemical applications requires control over channel activity. Herein we report a reversible control over the functioning of a mechanosensitive ion channel by interfering with its interaction with the lipid bilayer. The mechanosensitive channel of large conductance from Escherichia coli is reconstituted into liposomes and activated to its different sub-open states by titrating lysophosphatidylcholine (LPC) into the lipid bilayer. Activated channels are closed back by the removal of LPC out of the membrane by bovine serum albumin (BSA). Electron paramagnetic resonance spectra showed the LPC-dose-dependent gradual opening of the channel pore in the form of incrementally increasing spin label mobility and decreasing spin-spin interaction. A method to reversibly open and close mechanosensitive channels to distinct sub-open conformations during their journey from the closed to the fully open state enables detailed structural studies to follow the conformational changes during channel functioning. The ability of BSA to revert the action of LPC opens new perspectives for the functional studies of other membrane proteins that are known to be activated by LPC.

Keywords: MscL, reversible gating, mechanosensation, electron spin resonance spectroscopy, lysophosphatidylcholines, bovine serum albumin

\section{INTRODUCTION}

Elucidating the mechanisms by which ion channels function remains an ongoing challenge in physiology. Even though invaluable structural information is obtained from crystal structures, it is not always possible to derive the conformational transitions necessary for function from the static structures. Therefore, complementary approaches that give information about the dynamic conformers are needed.

We investigated a mechanosensitive ion channel (MSC) from Escherichia coli (E. coli). In nature, MSC's sense the changes in membrane tension and are involved in hearing, the sense of equilibrium, touch, pain, as well as renal and cardiovascular regulation (Blount et al., 2007). In E. coli, mechanosensitive channel of large conductance (MscL) protects cells from a sudden hypoosmotic shock in the external environment by opening a non-selective, temporary pore and releasing small solutes out of the cell (Sukharev et al., 1994). The channel is composed of five identical subunits (Chang et al., 1998; Steinbacher et al., 2007). The N- and C-terminus of the 
channel is in the cytoplasm, and each subunit has two transmembrane helices connected by a flexible periplasmic loop (Figure 1A). MscL directly translates the mechanical forces in the lipid bilayer into large conformational changes. It does not need any other cell components for its function (Blount and Moe, 1999; Koprowski and Kubalski, 2001). Furthermore, the crystal structures of MscL-homologs in their closed state exist but could not describe the mechanism of channel opening and closing, i.e., gating (Chang et al., 1998; Steinbacher et al., 2007). Recently, we showed that global conformational changes of MscL can be observed using ion mobility native mass spectrometry (Konijnenberg et al., 2014). However, to understand the onset of mechanosensation at the molecular level, there is a need for following the conformational changes from the start of channel opening and closing and relating these conformational changes to the forces in the lipid bilayer. Since the conformational changes leading to full channel opening start even before the channel pore starts conducting ions (Gullingsrud et al., 2001; Gullingsrud and Schulten, 2004), conventional patch clamp electrophysiology, which measures ionic current flowing through an open pore, would miss the initial events. Instead, spectroscopic techniques reporting on the conformational changes would be very useful.

One of the challenges of studying a mechanosensitive ion channel by spectroscopy is to design a tool to control the channel opening and closing. Here we show an approach to achieve this goal. In patch clamp electrophysiology, the membrane can be stretched by applying suction via the recording pipette in a controlled manner. However, this level of control over the channel activity in a spectroscopy setting is missing. To this end, pioneering works by Perozo et al. (2002a,b) explored the lipid bilayer mechanical properties. They showed that reconstituting MscL into lipids with different acyl chain lengths, hence changing the hydrophobic mismatch between the ion channel and the lipid bilayer, lowers the activation energy of MscL and traps the channel in structurally distinct closed intermediate states. On the other hand, asymmetric insertion of an inverted-coneshaped non-bilayer forming lipid L- $\alpha$-lysophosphatidylcholine (LPC) into one leaflet of a lipid bilayer (Perozo et al., 2002a,b; Yoshimura and Sokabe, 2010), fixes MscL in its open conformation. In another study, MscL activity was initiated by light in spectroscopic setups (Yilmaz et al., 2015). However, reversible control over channel activity is still missing.

Here, we present a tool that enables reversible activation of MscL in situ by LPC and BSA (Figure 1B). In cellular signal transduction, albumins are known to regulate LPC concentration under physiological conditions (Guo et al., 2009) by binding LPC molecules and facilitating their transport. One albumin molecule has a binding capacity of four to five LPC molecules (Thumser et al., 1994; Kim et al., 2007). Indeed, by employing the fluorescence and site-directed spin-label electron paramagnetic resonance (SDSL-EPR) spectroscopy, we show that while the incorporation of defined concentrations of LPC into the liposomes opens MscL into different sub-open states, some of which might not yet be conducting ions, the addition of BSA closes the channels back. Furthermore, based on the relation between the concentration of LPC and tension necessary for activation of MscL (Mukherjee et al., 2014), here we managed to activate MscL to distinct sub-open states by titrating the lipid bilayer with LPC.

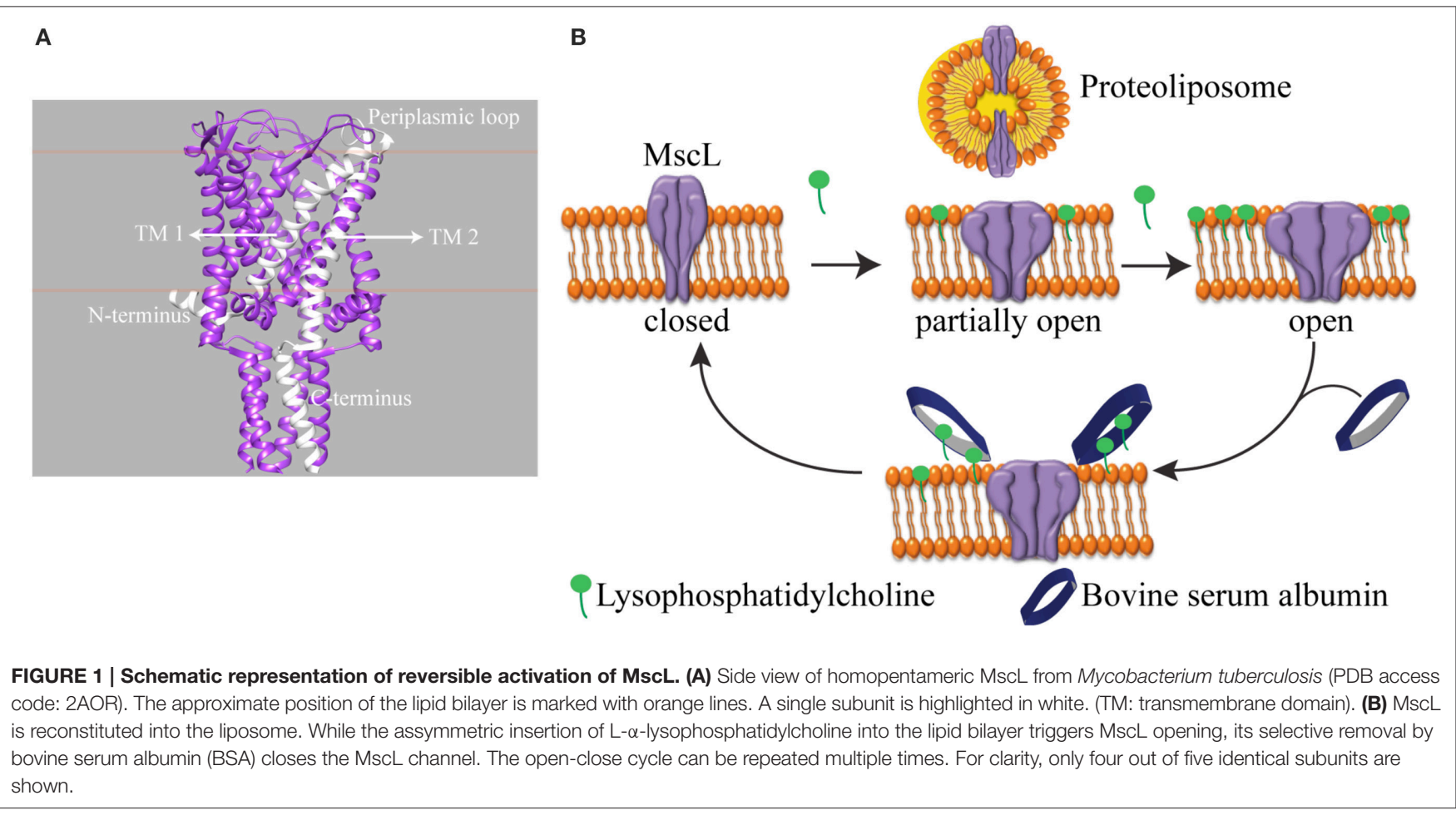


We anticipate that reversible control over MscL activity and reaching sub-open conformations by LPC titration will allow to investigate conformational changes of MscL from the onset of channel activation and provide a valuable tool to understand the tension-sensing mechanism of these channels. The findings will pave the way to study other membrane proteins that were shown to be activated by LPC (Marra et al., 2016). Beyond investigating the structure-function relation, technological applications such as biosensors (Kocer et al., 2012; Urban et al., 2014), environment-responsive drug delivery devices (Kocer et al., 2005, 2006; Kocer, 2007; PachecoTorres et al., 2015), or membrane-mediated protein-protein communication networks (Charalambous et al., 2012) could also benefit from the reversible control over the activity of such ion channels.

\section{MATERIALS AND METHODS}

\section{Materials}

All common chemicals were of reagent grade and were purchased from Sigma-Aldrich Chemie B. V., Netherlands. (1-oxyl-2,2,5,5tetramethylpyrroline-3-methyl) methanethiosulfonate (MTSSL) and Methoxypoly(ethyleneglycol)5000 Amidopropionyl Methanethio-sulfonate (MTS-PEG5000) were purchased from Toronto Research Chemicals Inc. (TRC), Toronto, Canada. Azolectin was from Avanti Polar Lipids. Polycarbonate membrane was from Avestin Europe, GmbH. Biobeads (SM-2 Absorbents) were from Bio-Rad Laboratories, Inc., CA, USA.

\section{Site-Directed Spin Labeling}

G22C mutant of MscL channels was produced and isolated as described before (Kocer et al., 2007). MTSSL was used as the spin-label. The purified MscL channels were labeled with MTSSL either maximally or minimally with MTSSL to protein $(\mathrm{mol} / \mathrm{mol})$ ratio of $5: 1$ or $1: 5(\mathrm{~mol} / \mathrm{mol})$ as explained before (Yilmaz et al., 2015). Briefly, labeling was performed by incubating the necessary amount of the spin label and detergent-solubilised MscL at room temperature for $30 \mathrm{~min}$ with gentle rotation. The labeling was stopped by removing the unbound MTSSL by Sephadex G-25 size exclusion column chromatography.

\section{Spin Labeling Efficiency}

The spin labeling efficiency (SLE) was calculated as explained before (Yilmaz et al., 2015). Briefly, the labeling was confirmed by the electrophoretic mobility shift assay (EMSA) and quantified by EPR. For the EMSA, the spin-labeled MscL (MscL-SL) was incubated for $5 \mathrm{~min}$ with $\beta$-mercaptoethanol-free SDS-PAGE sample buffer. This step dissociates MscL into its monomers and thereby exposes the free cysteines. Next, a cysteine-specific high molecular weight compound MTS-PEG5000 was added to the sample to a $2 \mathrm{mM}$ final concentration. Subsequently, the protein was separated on a $12.5 \%$ SDS/PAGE gel. For quantification, the gel was scanned using a Fujifilm LAS-3000 imager and was analyzed by using AIDA image analyzing software (Raytest $\mathrm{GmbH})$.

The SLE was quantified by comparing the integral of the EPR absorption spectra of the labeled protein and a reference spin probe MTSSL of known concentration by using Equation (1):

$$
C_{s a m p}=\frac{N_{s a m p} \cdot C_{r e f}}{N_{r e f}}
$$

Where, $\boldsymbol{C}_{\text {samp }}$ is the concentration of MTSSL in the labeled protein, $\boldsymbol{N}_{\text {samp }}$ and $\boldsymbol{N}_{\text {ref }}$ are the evaluated integrals of the absorption spectra of the sample and the reference, respectively, and $\boldsymbol{C}_{r e f}$ is the reference concentration. Typically, $100 \mathrm{mM}$ MTSSL dissolved in DMSO was used as a reference. Then, $C_{\text {samp }}$ was used to calculate the SLE by Equation (2):

$$
S L E=\frac{C_{\text {samp }}}{C_{\text {prot }}}
$$

Where, $C_{\text {prot }}$ is the concentration of the labeled protein determined by the standard Bradford assay.

\section{Reconstitution of MTSSL-Labeled G22C MscL into Liposomes}

The protein was reconstituted into liposomes as previously described (Kocer et al., 2007), with slight modifications. Briefly, $20 \mathrm{mg} / \mathrm{ml}$ azolectin in a lipid buffer $(150 \mathrm{mM} \mathrm{NaCl}, 10 \mathrm{mM}$ sodium phosphate buffer, $\mathrm{pH}$ 8.0) was subjected to five freeze-thaw cycles in liquid nitrogen and $50^{\circ} \mathrm{C}$, respectively. The liposomes were sized by extrusion through a $400 \mathrm{~nm}$ polycarbonate membrane. The resulting large unilamellar liposomes were saturated by the addition of final $9 \%(\mathrm{v} / \mathrm{v})$ detergent (Triton X-100). Labeled protein and detergentsaturated liposomes were mixed at 1:50 molar ratio and incubated for $30 \mathrm{~min}$ at $50^{\circ} \mathrm{C}$. Subsequently, either $10 \mathrm{mM}$ sodium phosphate buffer, $\mathrm{pH} 8.0$ (for EPR measurements) or $200 \mathrm{mM}$ calcein (for fluorescence measurements) was added in 1:1 volume ratio and supplemented with $6 \mathrm{mg}$ (wet weight) Biobeads (SM-2 Absorbents) per $1 \mu \mathrm{L}$ detergent (10\% Triton X100). For detergent removal, the sample was incubated overnight at $4^{\circ} \mathrm{C}$ under mild agitation.

For EPR experiments, proteoliposomes were concentrated by centrifugation at $135,000 \mathrm{~g}$ for $50 \mathrm{~min}$ at $4^{\circ} \mathrm{C}$ and resuspended in $20-50 \mu \mathrm{L}$ of $10 \mathrm{mM}$ sodium phosphate buffer, $\mathrm{pH}$ 8.0.

\section{Fluorescence Dequenching Assay}

Before the fluorescence dequenching experiments, the reconstituted calcein-loaded proteoliposomes were subjected to a size exclusion chromatography using Sephadex G50 matrix for separating the external calcein. The matrix was equilibrated with the efflux buffer $(10 \mathrm{mM}$ sodium phosphate buffer, $\mathrm{pH}=8 ; 150 \mathrm{mM} \mathrm{NaCl} ; 1 \mathrm{mM}$ EDTA). Next, a $2 \mu \mathrm{L}$ of proteoliposomes were added to a cuvette filled with $2100 \mu \mathrm{L}$ efflux buffer. Fluorescence emission was monitored at $515 \pm$ $2 \mathrm{~nm}$ (excitation at $495 \pm 2 \mathrm{~nm}$ ) in a Cary Eclipse Fluorescence Spectrophotometer (Varian Inc.). Channel activation at different LPC concentrations was achieved by the addition of LPC to the sample and followed for approximately $3 \mathrm{~min}$. Channel deactivation was achieved by adding BSA to already LPCcontaining sample and followed for $1 \mathrm{~min}$. Six different final LPC concentrations were tested: $1,1.5,2.0,2.5,3.0$, and $3.5 \mu \mathrm{M}$, 
whereas $25 \mathrm{~mol} \%$ BSA with respect to LPC concentration was added for channel deactivation. At the end of each experiment, proteoliposomes were lysed by the addition of Triton X100, and the resulting fluorescence value was taken as $100 \%$.

The release of calcein was calculated as a percentage of the total calcein amount present in the proteoliposomes:

$$
\% \text { Release }=\left[\left(\mathrm{I}-\mathrm{I}_{\mathrm{o}}\right) /\left(\mathrm{I}_{100}-\mathrm{I}_{0}\right)\right]^{*} 100
$$

Where, I is the measured fluorescence intensity at a given time, Io is the initial background fluorescence intensity, and $\mathrm{I}_{100}$ is the fluorescence intensity upon complete lysis of the proteoliposomes, which was elicited by adding $100 \mu \mathrm{L}$ Triton $\mathrm{X}-100$ from a $10 \%$ stock.

\section{EPR Spectroscopy}

Continuous wave (cw) EPR measurements were performed using a MiniScope benchtop X-band EPR spectrometer (MS400 Magnettech $\mathrm{GmbH}$, Berlin, Germany) with a rectangular TE102 resonator. The cavity was fluxed with gaseous nitrogen to keep the temperature stable. The microwave power and the Bfield modulation amplitude were set to $10 \mathrm{~mW}$ and $0.20 \mathrm{mT}$, respectively (see supplementary information for determination of overmodulation in this particular instrument). EPR glass capillaries with $0.9 \mathrm{~mm}$ inner diameter were filled with a sample volume of $10 \mu \mathrm{L}$ (for LPC) and $14 \mu \mathrm{L}$ (for BSA) at a final MscL concentration of 170-200 $\mu \mathrm{M}$.

The microwave frequency was $9.41 \mathrm{GHz}$; the modulation frequency was $100 \mathrm{kHz}$. Each spectrum of maximally labeled protein corresponds to the accumulation of 16 scans while spectra of minimally labeled protein correspond to the accumulation of 25 scans.

\section{Channel Activation and Deactivation Followed by EPR}

EPR experiments at increasing LPC concentrations were carried out from a single stock of spin labeled MscL (MscL$\mathrm{SL})$ reconstituted in liposomes. The stock was divided into six aliquots (including one control sample, i.e., labeled and reconstituted MscL-SL without LPC addition). A desired LPC to azolectin ratio was obtained by adding the required amount of LPC to a constant final volume $(10 \mu \mathrm{L})$ always $1 \mathrm{~h}$ before starting the EPR experiment. Channel closure was achieved by adding BSA to LPC-activated channels at $25 \mathrm{~mol} \%$ with respect to LPC concentration (LPC: BSA molar ratio) to $14 \mu \mathrm{L}$ final volume.

\section{Analysis of EPR Spectra}

To determine spin-label mobility the inverse peak-to-peak central linewidth $\Delta \mathrm{H}^{-1}$ and the second moment $<\Delta \mathrm{B}^{2}>$ were used. After base-line correction, using the MS400-Analysis software (Magnettech $\mathrm{GmbH}$, Berlin, Germany), the first and second moments of the EPR spectra were calculated numerically using the UNISPC program (provided by Dr. Johann Klare, Universität Osnabrück, Germany).
The difference in spectral second moment $\Delta<\Delta \mathrm{B}^{2}>$ was calculated according to the following equation (Steinhoff, 2002):

$$
\begin{aligned}
\Delta<\Delta \mathrm{B}^{2}> & =<\Delta \mathrm{B}_{I}^{2}>-<\Delta \mathrm{B}_{N}^{2}> \\
& =\frac{\int\left(B-B_{F I}\right)^{2} S_{I}(B) d B}{\int S_{I}(B) d B}-\frac{\int\left(B-B_{F N}\right)^{2} S_{N}(B) d B}{\int S_{N}(B) d B}
\end{aligned}
$$

Where $<\Delta \mathrm{B}_{I}^{2}>$ and $<\Delta \mathrm{B}_{N}^{2}>$ are second moments of dipolar-broadened (interacting spins) and non-broadened (noninteracting spins) EPR spectra, respectively; $S_{I}(B)$ is the EPR absorption spectrum in which the dipolar spin-spin interaction is present, whereas $S_{N}(B)$ is the spectrum without spin-spin interaction; $B_{F I}$ and $B_{F N}$ are the first spectral moments of the respective spectra, and $B$ is the static magnetic field.

\section{RESULTS}

\section{MscL Can Be Reversibly Activated in Liposomes}

First, to demonstrate the action of LPC and BSA on MscL, we performed a fluorescence dequenching assay using MscL reconstituted into liposomes (Kocer et al., 2007). Insertion of LPC into the outer bilayer leaflet was achieved by adding a defined concentration of LPC to freshly prepared G22C MscL proteoliposomes that were loaded with a self-quenching fluorescent dye, calcein. The dye is released through MscL channels and the concomitant increase in fluorescence is followed in time. Figure 2 shows that upon the addition of 40

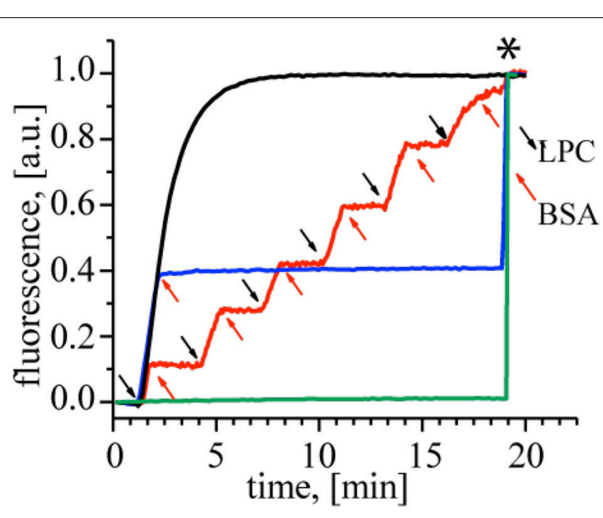

FIGURE 2 | Reversible activation of MscL by LPC and BSA. Black trace: Calcein loaded control MscL proteoliposomes treated with 40 mol\% LPC reaches maximum calcein release within $5 \mathrm{~min}$. When the duplicate of the control sample was treated with 25 mol\% BSA (relative to LPC concentration) at $t=2 \mathrm{~min}$, the calcein release stopped immediately (b/ue trace). The red trace shows repeated opening and closing cycles of MscL. Consecutive LPC and BSA additions indicated by black and red arrows, respectively. Calcein-loaded liposomes with no MscL channel were used as a negative control to test the effect of $40 \mathrm{~mol} \%$ LPC (relative to the lipid concentration) and 25 mol\% BSA (relative to LPC) on the lipid bilayer itself (green trace), showing that the indicated amounts of LPC and BSA did not cause any leakage through the lipid bilayer. The asterisk marks the time point of detergent addition to the samples for lysing the liposomes and releasing all calcein. 
mol\% LPC (relative to lipid concentration), the calcein release reaches a plateau within $5 \mathrm{~min}$ (black line). If, on the other hand, BSA ( 25 mol\% with respect to LPC concentration) was added to a duplicate of the first sample before the release reaches the plateau, the release stopped immediately (Figure 2, blue line), indicating that LPC and BSA can be used to open and close the channel, respectively. We showed that the cycle of opening and closing of the channel could be repeated multiple times, as shown with the red line in Figure 2. Together, the results show a lipid-mediated reversible control of $\mathrm{MscL}$ gating.

\section{MscL Requires Less than $10 \mathrm{~mol} \%$ LPC for Its Activation}

Next, we tested reversible activation of MscL by employing EPR spectroscopy. In our experiments, we monitored the mobility of a pore amino acid of MscL upon activation and deactivation by LPC-BSA. To perform the EPR experiments, the cysteine at the 22nd position of MscL (see Figure 3A) was labeled either maximally or minimally with ((1-oxyl-2,2,5,5tetramethylpyrrolin-3-methyl) methanethiosulfonate) (MTSSL) (Altenbach et al., 1989). The choice of the G22 position is based on its critical location in the pore (Yoshimura et al., 1999), its high accessibility for labeling (Kocer et al., 2005, 2006), and the significant increase in spin-label mobility at this site upon channel activation (Perozo et al., 2002a,b). The MTSSL labeling of MscL was confirmed by an EMSA (see Supplementary Figure 1) and quantified by employing EPR spectroscopy. The SLE for the maximally and minimally labeled samples were 88 and $18 \%$, respectively.

After showing that LPC has no effect on detergent solubilized MscL (Figure 3B, top panel), the channels were reconstituted into liposomes and used for EPR measurements. The black line in the middle and bottom panel of Figure 3B shows the spectra of the maximally labeled channel in its initial closed state. Addition of $40 \mathrm{~mol} \%$ LPC relative to the lipid concentration (opening of the channel) significantly narrowed the line width of the EPR spectrum (Figure 3B, middle panel, red line). Increase of the LPC concentration above $40 \mathrm{~mol} \%$ did not change the EPR spectrum anymore. The addition of $25 \mathrm{~mol} \% \mathrm{BSA}$ (relative to LPC concentration), on the other hand, generated a signal that completely reverses the changes seen in the middle panel and results in a spectrum that is very similar to that of the closed MscL channel (Figure 3B, lower panel, blue line).

After showing that 40 mol\% LPC opens the channel fully, and that the EPR spectra differ significantly for the closed and the open channel, we set out to determine, if intermediate opening steps of the MscL channel can be reached by adding LPC at concentrations below $40 \%$. Indeed, EPR line shape changes can be observed at concentrations as low as $10 \mathrm{~mol} \%$. Between 10 and $30 \mathrm{~mol} \%$ LPC the EPR line shape changes continuously, and the $30 \mathrm{~mol} \%$ spectrum is identical to the one at $40 \mathrm{~mol} \% \mathrm{LPC}$ (Figure 3C).

\section{Channel Opening Monitored by EPR}

The EPR spectra of maximally and minimally labeled MscL samples (Figure 4A, black and red, respectively) revealed a narrowing of the spectral lines with increasing LPC concentration. In addition, the hallmarks of the slow rotation in nitroxide EPR spectra, as marked by arrows in Figure 4A on the high and low field side of the spectra, diminished and finally disappeared. Between 20 and $30 \mathrm{~mol} \%$ of LPC, especially the spectra of the maximally labeled sample change, whereas those of the minimally labeled one are almost identical.

To quantitate the changes in EPR line-shape, two mobility parameters, inverse central line width $\left(\Delta \mathrm{H}^{-1}\right)$ and second moment $\left(<\Delta B^{2}>\right)$, were calculated from the EPR spectra of the minimally labeled sample. The inverse central line width increases as the mobility of the spin label increases, since less mobility causes broadening of the EPR lines. The second moment (Slichter, 1992) is a measure of the overall width of the EPR spectrum that can be determined for a series of spectra also in the presence of overall line shape changes, so it is a robust measure of the broadening. Also common in the literature is to use the inverse second moment as a measure of mobility, as introduced by the Hubbell group (Mchaourab et al., 1996). In Figure 4B these parameters are plotted as a function of LPC concentration. Both parameters show the increase in spin-label mobility up to LPC concentrations of $30 \mathrm{~mol} \%$ and then remain constant within the experimental error (see Figure 4B). Together, these results show that the spin-label mobility increases as the LPC concentration increases.

The second trend manifests itself in a broadening of the EPR spectra of the maximally labeled (Figure 4A, black) with respect to the minimally labeled (Figure 4A, red) EPR spectra LPC concentrations up to $30 \%$ of LPC. This is most obvious in the spectrum with $20 \mathrm{~mol} \% \mathrm{LPC}$ at field values around $333 \mathrm{mT}$ (Figure 4A, the left arrow).

Line broadening in the maximally labeled with respect to the minimally labeled sample suggests that there is a spin-spin interaction in the maximally labeled sample when the channel is in its closed state (i.e., at low LPC concentrations, i.e., $\leq 10 \%$ ). However, as the LPC concentration increases, the spin-spin interaction between spin labels attached to different subunits of $\mathrm{MscL}$ reduces, suggesting pore opening. A measure for the amount of broadening of the EPR spectra is the difference in spectral second moments (Equation 4 ), $\Delta<\Delta \mathrm{B}^{2}>$, the values of which are given in Table $\mathbf{1}$.

Although overall small, the $\Delta<\Delta \mathrm{B}^{2}>$ values show a sharp decrease between 20 and 30\% LPC concentration suggestive of channel opening (for details, see Discussion). The difference in second moment values at and above $30 \mathrm{~mol} \%$ LPC are so small, that spin-spin interaction is considered to be negligible.

\section{DISCUSSION}

In the present study, we could reversibly control MscL activity by exploring the LPC-BSA interplay on the lipid bilayer. The only other method allowing reversible control on this channel in situ was a reversible light switch, which was covalently linked to the pore region of MscL (Kocer et al., 2005). However, spectroscopy probes, such as MTSSL, also attach to the protein via cysteine chemistry, which complicates the channel labeling (Yilmaz et al., 2015). 


\section{A}

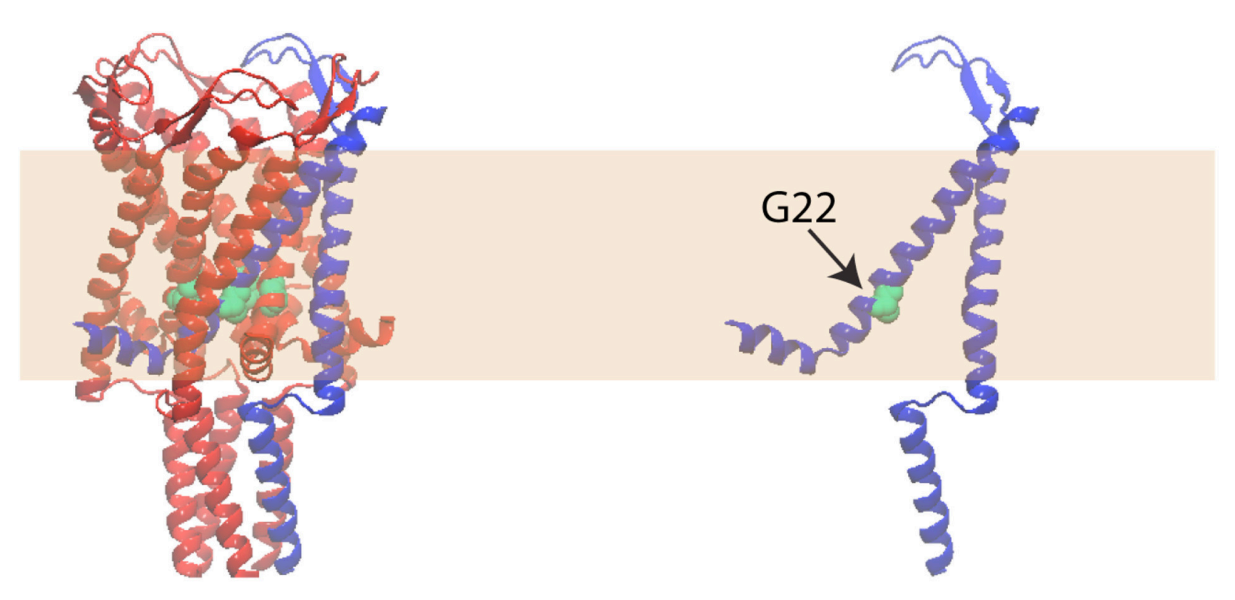

B
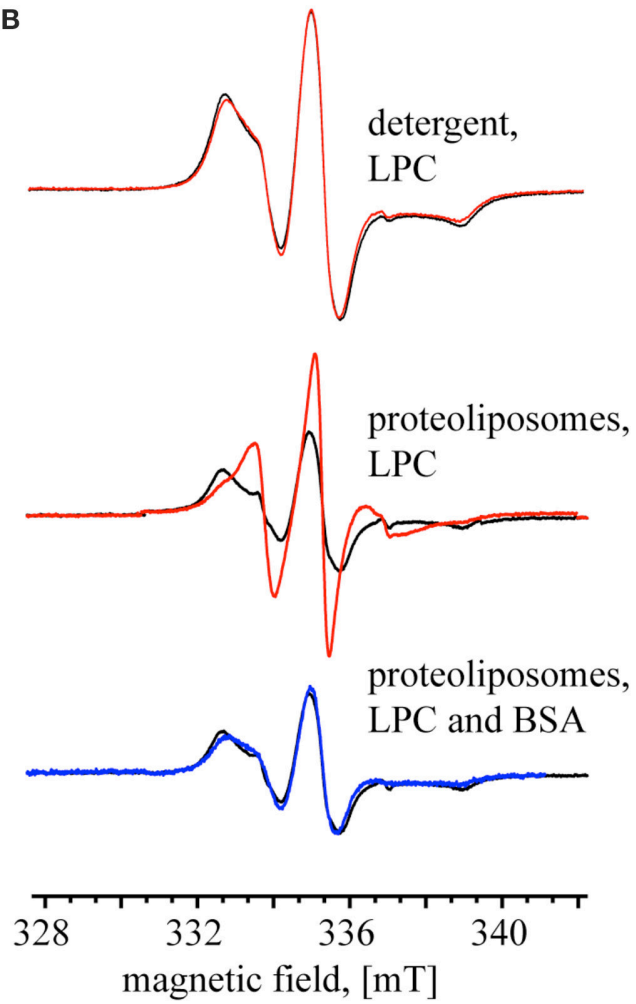

C

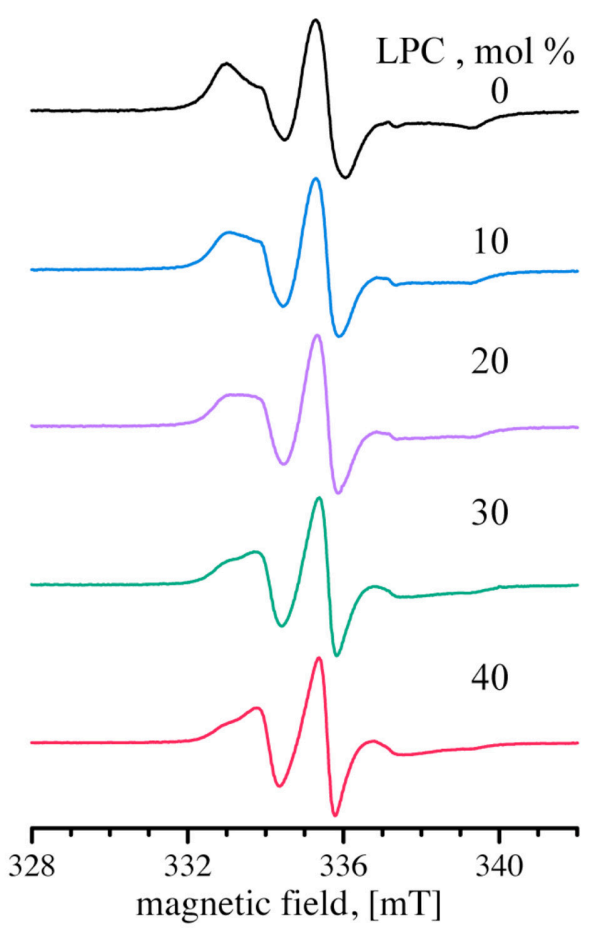

FIGURE 3 | Reversible activation of MTSSL-labeled G22C MscL monitored by continuous wave EPR. (A) Schematic representation of MscL based on the crystal structure of the homologous MscL from Mycobacterium tuberculosis (PDB access code: 2AOR). The yellow area represents the lipid bilayer. (B) MscL channel activation by 40 mol\% LPC (relative to the lipid concentration). Top and Middle: the spectra show MTSSL-labeled G22C MscL (MscL-SL, minimally labeled) in the absence of LPC (black); activated with LPC (red). Bottom: the spectra of LPC-activated channels after adding BSA to the proteoliposomes (b/ue). Spectra are normalized to the same number of spins. (C) EPR spectra of maximally labeled MscL-SL samples with increasing mol\% of LPC, percentages are given relative to the lipid concentration. The magnetic field scale shown at the bottom of $\mathbf{( B , C )}$ refers to all spectra of the respective set.

With the present approach, we showed the feasibility of titrating MscL channel opening to different sub-open states by dose-dependent activation of the channel by LPC. Previously, high concentrations of LPC has been used to open MscL completely to study the conformational changes of the pore forming TM1 helix (Perozo et al., 2002a,b) and, recently, the $\mathrm{N}$-terminus of MscL (Bavi et al., 2016). Interestingly, despite its dramatic effect on the channel opening, the mechanism of LPC-induced channel activation is still debated (Yoshimura and Sokabe, 2010). The partitioning of LPC changes the lipid bilayer properties (Zhelev, 1998) some of which have been proposed as the mechanism of LPC effect on MscL. such as changes in the lateral pressure profile (Perozo et al., 2002a; Esteban-Martín et al., 2009), bending of the membrane 
A

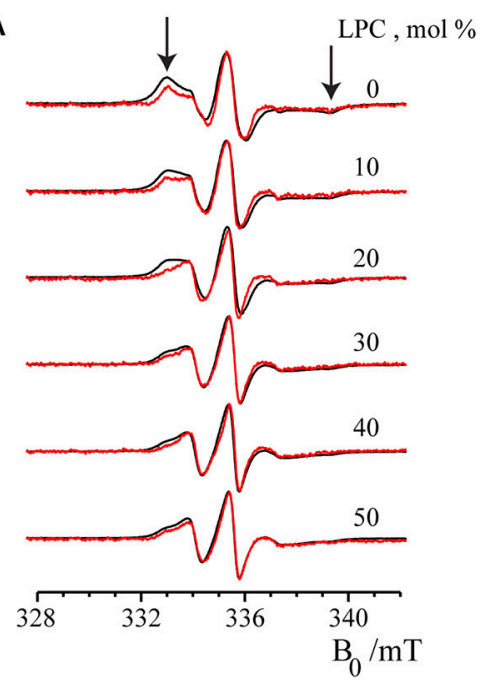

B

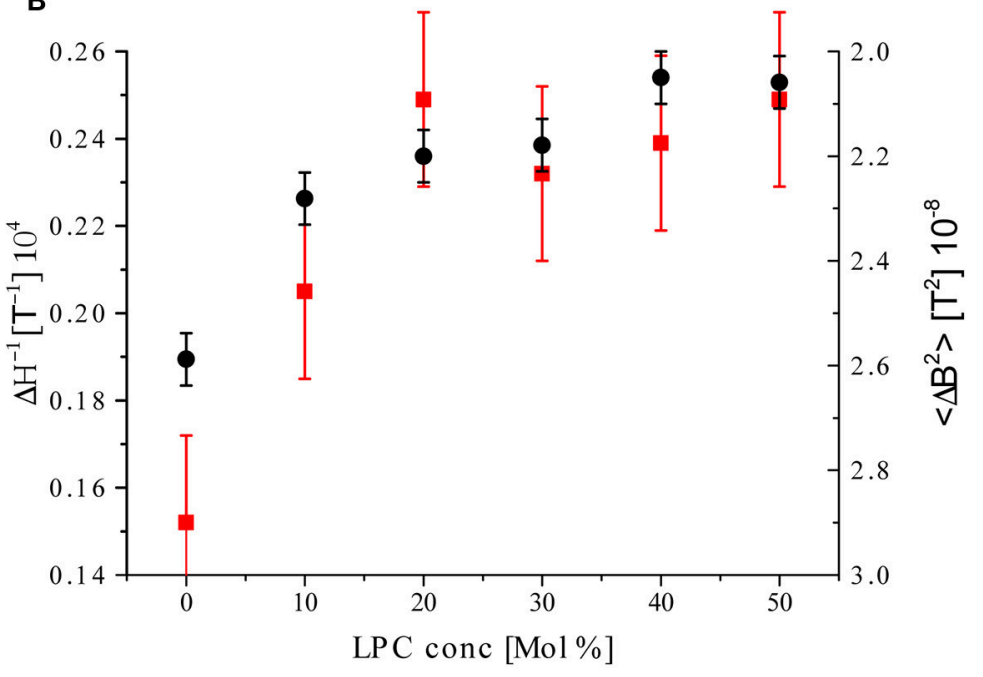

FIGURE 4 | Room temperature cw EPR spectra of minimally labeled and maximally labeled MscL-SL. (A) Black: maximally labeled, red: minimally labeled MscL. LPC concentrations in the liposomes are indicated. Spectra are normalized to the amplitude of the central line. The arrows indicate features of the spectra that derive from spin labels that are slowly rotating (see text). The features indicated by arrows diminish and disappear upon increasing the concentration of LPC. (B) Parameters describing spin label mobility as a function of LPC concentrations: Inverse central line width $\left(\Delta \mathrm{H}^{-1}\right.$, red squares) and second moment $\left(<\Delta \mathrm{B}^{2}\right\rangle$, black dots) of the minimally labeled sample. Increased mobility is reflected in a narrower line, which results in a larger inverse central line width $\left(\Delta \mathrm{H}^{-1}\right)$, and a smaller second moment $\left(<\Delta \mathrm{B}^{2}>\right)$ parameter.

\begin{tabular}{|c|c|}
\hline $\begin{array}{l}\text { LPC conc. } \\
\text { [mol\%] }\end{array}$ & $\begin{array}{l}\Delta<\Delta B^{2}>\times 10^{-10} \\
{\left[T^{2}\right] \pm 20 \times 10^{-10}}\end{array}$ \\
\hline $0^{*}$ & 99 \\
\hline 10 & 146 \\
\hline 20 & 145 \\
\hline 30 & 84 \\
\hline 40 & 98 \\
\hline 50 & 82 \\
\hline
\end{tabular}

Line broadening is expressed as the second moment difference $\Delta<\Delta B^{2}>$ in $T^{2}$ obtained from spectra of maximally and minimally labeled MscL.

*The second moment of the maximally labeled sample is smaller than expected considering the values at 10 and $20 \%$ LPC, possibly due to a not fully closed channel in this particular sample, resulting in a too small $\Delta<\Delta B^{2}>$ value.

(Wiggins and Phillips, 2004), and changes in the surface tension (Yoshimura and Sokabe, 2010). In a recent work, we have shown that LPC does not interact with MscL to exert its effect but requires the lipid bilayer. However, its action cannot solely be explained by the changes in the lipid bilayer properties (Mukherjee et al., 2014), either. While the amount of LPC partitioning in a liposome would mimic the magnitude of applied tension in the patch clamp by discriminating tension-sensitive mutants of MscL from E. coli, it failed in distinguishing MscL from E. coli, Mycobacterium tuberculosis, and Lactococcus lactis, based on their tensionsensitivity. Therefore, it has been suggested that LPC exerts its effect on the MscL by changing the protein-membrane coupling, as also proposed by others (Lee, 2004; Marsh, 2008; Lundbaek et al., 2010).

To ensure maximum sensitivity of the EPR spectra to the changes occurring at different sub-open states, we designed the study to be sensitive to two properties: the spin label mobility and the spin-spin interaction, similar to approaches presented earlier (Der-Sarkissian et al., 2003; Margittai and Langen, 2006; Scarpelli et al., 2009). The mobility of the spin label at channel position 22 increases stepwise and in a dose dependent way, with increasing concentrations of LPC, showing stepwise opening of the channel (see Figures 1, 3A, 4B). As the channel opens, its pore widens, providing more space for the spin label to move. The observed mobility increase is in agreement with previous EPR studies on the fully open channel (Perozo et al., 2002a,b).

Similarly, the spin-spin interaction, which depends on the distance between the spin labels, decreases, showing an increase in the distance between the spin labels as the LPC concentration increases. Again this decrease in spin-spin interaction is expected as the channel opens, since the widening of the pore increases the distance between the protein subunits to which the spin labels are attached. Quantification of the increase in distance (Steinhoff, 2002) between the subunits, and thereby structural information of the channel in different open states, cannot be obtained under the conditions of the present study, because of the labeling conditions employed. In the Supplementary Material we give a detailed account of why distances cannot be extracted and describe how specific assembly of channels, as described in Birkner et al. (2012) would solve this issue.

Together with the ability to open the channel to intermediate states, our approach allows to close the channel from any open 
state back to the closed conformation. This will enable detailed structural studies in the future and will provide a more complete picture of the gating mechanism of MscL from the onset of the mechanosensation.

\section{AUTHOR CONTRIBUTIONS}

$\mathrm{MW}, \mathrm{AD}$, and $\mathrm{AK}$ designed the research, MW, $\mathrm{AD}$ performed research, $\mathrm{PK}$ and $\mathrm{MHS}$ performed experiments and provided analysis methodology, MW, AD, MH, AK analyzed data, and all authors contributed to writing the article.

\section{FUNDING}

Financial support by the Foundation for Fundamental Research on Matter (FOM), which is part of the Netherlands Organisation

\section{REFERENCES}

Altenbach, C., Flitsch, S. L., Khorana, H. G., and Hubbell, W. L. (1989). Structural studies on transmembrane proteins. 2. Spin labeling of bacteriorhodopsin mutants at unique cysteines. Biochemistry 28, 7806-7812. doi: 10.1021/ bi00445a042

Bavi, N., Cortes, D. M., Cox, C. D., Rohde, P. R., Liu, W., Deitmer, J. W., et al. (2016). The role of MscL amphipathic $\mathrm{N}$ terminus indicates a blueprint for bilayer-mediated gating of mechanosensitive channels. Nat. Commun. 7:11984. doi:10.1038/ncomms11984

Birkner, J. P., Poolman, B., and Koçer, A. (2012). Hydrophobic gating of mechanosensitive channel of large conductance evidenced by single-subunit resolution. Proc. Natl Acad. Sci. U.S.A. 109, 12944-12949. doi: 10.1073/ pnas. 1205270109

Blount, P., Iscla, I., Moe, P. C., and Li, Y. (2007). "MscL: the bacterial mechanosensitive channel of large conductance," in Current Topics in Membranes, Vol. 58, ed O. P. Hamill (Texas: Elsevier), 201-233.

Blount, P., and Moe, P. C. (1999). Bacterial mechanosensitive channels: integrating physiology, structure and function. Trends Microbiol. 7, 420-424. doi: 10.1016/S0966-842X(99)01594-2

Chang, G., Spencer, R. H., Lee, A. T., Barclay, M. T., and Rees, D. C. (1998). Structure of the MscL homolog from Mycobacterium tuberculosis: a gated mechanosensitive ion channel. Science 282, 2220-2226. doi: 10.1126/ science.282.5397.2220

Charalambous, K., Booth, P. J., Woscholski, R., Seddon, J. M., Templer, R. H., Law, R. V., et al. (2012). Engineering de novo membrane-mediated protein-protein communication networks. J. Am. Chem. Soc. 134, 5746-5749. doi: 10.1021/ ja300523q

Der-Sarkissian, A., Jao, C. C., Chen, J., and Langen, R. (2003). Structural organization of alpha-synuclein fibrils studied by site-directed spin labeling. J. Biol. Chem. 278, 37530-37535. doi: 10.1074/jbc.M305266200

Esteban-Martín, S., Risselada, H. J., Salgado, J., and Marrink, S. J. (2009). Stability of asymmetric lipid bilayers assessed by molecular dynamics simulations. J. Am. Chem. Soc. 131, 15194-15202. doi: 10.1021/ja904450t

Gullingsrud, J., Kosztin, D., and Schulten, K. (2001). Structural determinants of MscL gating studied by molecular dynamics simulations. Biophys. J. 80, 2074-2081. doi: 10.1016/S0006-3495(01)76181-4

Gullingsrud, J., and Schulten, K. (2004). Lipid bilayer pressure profiles and mechanosensitive channel gating. Biophys. J. 86, 3496-3509. doi: 10.1529/biophysj.103.034322

Guo, S., Shi, X., Yang, F., Chen, L., Meehan, E. J., Bian, C., et al. (2009). Structural basis of transport of lysophospholipids by human serum albumin. Biochem. J. 423, 23-30. doi: 10.1021/bi061657l

Kim, Y. L., Im, Y. J., Ha, N. C., and Im, D. S. (2007). Albumin inhibits cytotoxic activity of lysophosphatidylcholine by direct binding. Prostaglandins Other Lipid Mediat. 83, 130-138. doi: 10.1016/j.prostaglandins.2006.10.006 for Scientific Research (NWO) (to $\mathrm{MH}$ ) and by European Research Council (Starting Grant 208814 to AK) is gratefully acknowledged.

\section{ACKNOWLEDGMENTS}

Dr. Johann Klare, Universität Osnabrück, Germany is acknowledged for providing us with UNISPC program for second moment calculations. We thank Prof. Michel Orrit for crucial help on probability distributions.

\section{SUPPLEMENTARY MATERIAL}

The Supplementary Material for this article can be found online at: http://journal.frontiersin.org/article/10.3389/fphys. 2016.00409

Kocer, A. (2007). A remote controlled valve in liposomes for triggered liposomal release. J. Liposome Res. 17, 219-225. doi: 10.1080/08982100701528203

Kocer, A., Tauk, L., and Déjardin, P. (2012). Nanopore sensors: from hybrid to abiotic systems. Biosens. Bioelectron. 38, 1-10. doi: 10.1016/j.bios.2012. 05.013

Koçer, A., Walko, M., Bulten, E., Halza, E., Feringa, B. L., and Meijberg, W. (2006). Rationally designed chemical modulators convert a bacterial channel protein into a pH-sensory valve. Angew. Chem. Int. Ed. Engl. 45, 3126-3130. doi: 10.1002/anie.200503403

Koçer, A., Walko, M., and Feringa, B. L. (2007). Synthesis and utilization of reversible and irreversible light-activated nanovalves derived from the channel protein MscL. Nat. Protoc. 2, 1426-1437. doi: 10.1038/nprot.2007.196

Koçer, A., Walko, M., Meijberg, W., and Feringa, B. L. (2005). A lightactuated nanovalve derived from a channel protein. Science 309, 755-758. doi: $10.1126 /$ science. 1114760

Konijnenberg, A., Yilmaz, D., Ingólfsson, H. I., Dimitrova, A., Marrink, S. J., Li, Z., et al. (2014). Global structural changes of an ion channel during its gating are followed by ion mobility mass spectrometry. Proc. Natl. Acad. Sci. U.S.A. 111, 17170-17175. doi: 10.1073/pnas.1413118111

Koprowski, P., and Kubalski, A. (2001). Bacterial ion channels and their eukaryotic homologues. Bioessays 23, 1148-1158. doi: 10.1002/bies.10017

Lee, A. G. (2004). How lipids affect the activities of integral membrane proteins Biochim. Biophys. Acta 1666, 62-87. doi: 10.1016/j.bbamem.2004.05.012

Lundbaek, J. A., Collingwood, S. A., Ingólfsson, H. I., Kapoor, R., and Andersen, O. S. (2010). Lipid bilayer regulation of membrane protein function: gramicidin channels as molecular force probes. J. R. Soc. Interface 7, 373-395. doi: 10.1098/rsif.2009.0443

Margittai, M., and Langen, R. (2006). "Spin labeling analysis of amyloids and other protein aggregates," in Amyloid, Prions, and Other Protein Aggregates, Part C Methods in Enzymology, eds R. Wetzel and I. Kheterpal (Elsevier), 122-139.

Marra, S., Ferru-Clément, R., Breuil, V., Delaunay, A., Christin, M., Friend, V., et al. (2016). Non-acidic activation of pain-related acid-sensing ion channel 3 by lipids. EMBO J. 35, 414-428. doi: 10.15252/embj.201592335

Marsh, D. (2008). Protein modulation of lipids, and vice-versa, in membranes Biochim. Biophys. Acta 1778, 1545-1575. doi: 10.1016/j.bbamem.2008.01.015

Mchaourab, H. S., Lietzow, M. A., Hideg, K., and Hubbell, W. L. (1996). Motion of spin-labeled side chains in T4 lysozyme. Correlation with protein structure and dynamics. Biochemistry 35, 7692-7704. doi: 10.1021/bi960482k

Mukherjee, N., Jose, M. D., Birkner, J. P., Walko, M., Ingolfsson, H. I., Dimitrova, A., et al. (2014). The activation mode of the mechanosensitive ion channel, MscL, by lysophosphatidylcholine differs from tension-induced gating. FASEB J. 28, 4292-4302. doi: 10.1096/fj.14-251579

Pacheco-Torres, J., Mukherjee, N., Walko, M., López-Larrubia, P., Ballesteros, P., Cerdán, S., et al. (2015). Image guided drug release from $\mathrm{pH}$-sensitive Ion channel-functionalized stealth liposomes into an in vivo glioblastoma model. Nanomedicine 11, 1345-1354. doi: 10.1016/j.nano.2015.03.014 
Perozo, E., Cortes, D. M., Sompornpisut, P., Kloda, A., and Martinac, B. (2002a). Open channel structure of MscL and the gating mechanism of mechanosensitive channels. Nature 418, 942-948. doi: 10.1038/nature00992

Perozo, E., Kloda, A., Cortes, D. M., and Martinac, B. (2002b). Physical principles underlying the transduction of bilayer deformation forces during mechanosensitive channel gating. Nat. Struct. Biol. 9, 696-703. doi: $10.1038 /$ nsb827

Scarpelli, F., Drescher, M., Rutters-Meijneke, T., Holt, A., Rijkers, D. T. S., Killian, J. A., et al. (2009). Aggregation of transmembrane peptides studied by spin-label EPR. J. Phys. Chem. B 113, 12257-12264. doi: 10.1021/jp901371h

Slichter, C. P. (ed.). (1992). "Magnetic dipolar broadening of rigid laticces," in Principles of Magnetic Resonance, Berlin: Springer, 65-85.

Steinbacher, S., Bass, R., and Strop, P. (2007). Structures of the prokaryotic mechanosensitive channels MscL and MscS. Curr. Top. Membr. 58, 1-24. doi: 10.1016/S1063-5823(06)58001-9

Steinhoff, H.-J. (2002). Methods for study of protein dynamics and protein-protein interaction in protein-ubiquitination by electron paramagnetic resonance spectroscopy. Front. Biosci. 7, c97-c110. doi: 10.2741/a763

Sukharev, S. I., Blount, P., Martinac, B., Blattner, F. R., and Kung, C. (1994). A large-conductance mechanosensitive channel in E. coli encoded by MscL alone. Nature 368, 265-268. doi: 10.1038/368265a0

Thumser, A. E., Voysey, J. E., and Wilton, D. C. (1994). The binding of lysophospholipids to rat liver fatty acid-binding protein and albumin. Biochem. J. 301(Pt 3), 801-806. doi: 10.1042/bj3010801

Urban, M., Kleefen, A., Mukherjee, N., Seelheim, P., Windschiegl, B., Vor der Brüggen, M., et al. (2014). Highly parallel transport recordings on a membraneon-nanopore chip at single molecule resolution. Nano Lett. 14, 1674-1680. doi: $10.1021 / \mathrm{nl} 5002873$
Wiggins, P., and Phillips, R. (2004). Analytic models for mechanotransduction: gating a mechanosensitive channel. Proc. Natl. Acad. Sci. U.S.A. 101, 4071-4076. doi: 10.1073/pnas.0307804101

Yilmaz, D., Dimitrova, A. I., Walko, M., and Kocer, A. (2015). Study of lightinduced MscL gating by EPR spectroscopy. Eur. Biophys. J. 44, 557-565. doi: 10.1007/s00249-015-1063-4

Yoshimura, K., Batiza, A., Schroeder, M., Blount, P., and Kung, C. (1999). Hydrophilicity of a single residue within MscL correlates with increased channel mechanosensitivity. Biophys. J. 77, 1960-1972. doi: 10.1016/S00063495(99)77037-2

Yoshimura, K., and Sokabe, M. (2010). Mechanosensitivity of ion channels based on protein-lipid interactions. J. R. Soc. Interface 7(Suppl. 3), S307-S320. doi: 10.1098/rsif.2010.0095.focus

Zhelev, D. V. (1998). Material property characteristics for lipid bilayers containing lysolipid. Biophys. J. 75, 321-330. doi: 10.1016/S0006-3495(98) 77516-2

Conflict of Interest Statement: The authors declare that the research was conducted in the absence of any commercial or financial relationships that could be construed as a potential conflict of interest.

Copyright (c) 2016 Dimitrova, Walko, Hashemi Shabestari, Kumar, Huber and Kocer. This is an open-access article distributed under the terms of the Creative Commons Attribution License (CC BY). The use, distribution or reproduction in other forums is permitted, provided the original author(s) or licensor are credited and that the original publication in this journal is cited, in accordance with accepted academic practice. No use, distribution or reproduction is permitted which does not comply with these terms. 